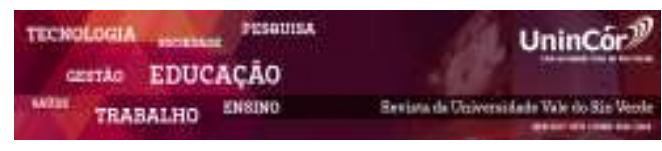
Revista da Universidade Vale do Rio Verde
ISSN: 1517-0276 / EISSN: 2236-5362
Vol. 16 | n. 2 | Ano 2018

Ellen Maria Neves Silva

Universidade Federal dos Vales do Jequinhonha e Mucuri (UFVJM) ellendtna@gmail.com

Paulo César de Resende Andrade Universidade Federal dos Vales do Jequinhonha e Mucuri (UFVJM) paulo.andrade@ict.ufvjm.edu.br

\section{ANÁLISE DE CONFIABILIDADE DE UM INSPETOR ELETRÔNICO DE GARRAFAS}

\section{RESUMO}

A confiabilidade de um equipamento está associada ao comportamento de falhas em equipamentos. O objetivo deste trabalho foi realizar uma análise da confiabilidade de um inspetor eletrônico de garrafas por meio da modelagem dos tempos até a falha. O procedimento de pesquisa utilizado foi o estudo de caso. Foram disponibilizados quarenta e três dados referentes a falhas ocorridas em dispositivos transportadores pertencentes ao inspetor. Métodos gráficos e analíticos foram utilizados para encontrar a distribuição que melhor modela o conjunto de dados. A análise foi feita utilizando o software ProConf. Os testes indicaram a distribuição Weibull como a que melhor se ajustou aos dados, com parâmetros de forma $\gamma$ igual a 1,64 e escala $\theta$ igual 3,93, determinados pelo método da verossimilhança. O tempo médio até a falha foi de 3,50 minutos. O risco crescente e a confiabilidade decrescente, associados ao fato do $\gamma$ ser maior que um, indicam um desgaste do equipamento. Isso corresponde ao início da mortalidade senil, fase final de sua vida útil. A estratégia para esta fase é a manutenção preventiva: a troca antecipa a inevitável quebra.

Palavras-chave: Confiabilidade. Equipamento. Comportamento de Falhas. Modelagem. Tempo de Vida.

\section{ANALYSIS OF RELIABILITY OF AN ELECTRONIC BOTTLE INSPECTOR}

\begin{abstract}
The reliability of an equipment is associated with the behavior of equipment failures. The objective of this work was to perform a reliability analysis of an electronic bottle inspector through timeto-failure modeling. The research procedure used was the case study. Forty-three data concerning failures occurred on conveyors belonging to the inspector were made available. Graphical and analytical methods were used to find the distribution that best models the data set. The analysis was done using ProConf software. The tests indicated the Weibull distribution as the best fit to the data, with parameters of form $\gamma$ equal to 1.64 and scale $\theta$ equal to 3.93 , determined by the likelihood method. The mean time to failure was 3.50 minutes. The increasing risk and decreasing reliability, associated with the fact that $\gamma$ is greater than one, indicates a wear of the equipment. This corresponds to the onset of senile mortality, the final phase of its useful life. The strategy for this phase is preventive maintenance: the exchange anticipates the inevitable break.
\end{abstract}

Keywords: Reliability. Equipment. Fault Behavior. Modeling. Lifetime.

Recebido em: 02/02/2018 - Aprovado em: 25/07/2018 - Disponibilizado em: 15/12/2018 


\section{INTRODUÇÃO}

O desenvolvimento tecnológico crescente, a alta competição global acirrada pela abertura de mercado e o aumento das expectativas do cliente por melhor desempenho em um período de tempo maior, têm impulsionado as indústrias a realizar grandes esforços com o intuito de fornecer produtos com melhor qualidade e mais confiáveis (DUEK, 2005).

Neste contexto, com a economia globalizada, surge uma intensa procura por equipamentos capazes de manter sua disponibilidade com mínimas probabilidades de falhas, resultando em bons investimentos a um menor custo de manutenção. As falhas em equipamentos ou sistemas causam efeitos que vão desde inconveniências menores até impactos severos no mercado, gerando perdas econômicas significativas (MENDES, 2011).

Em consequência, Marcorin e Abackerli (2001, p. 2) destacam que "o domínio da confiabilidade nos produtos confere à empresa uma vantagem competitiva em relação aos seus concorrentes, que se traduz, na prática, na melhor alocação de custos de garantia e de suporte, inventário de peças de reposição mais adequado, e menor custo estendido de seus produtos ao longo do ciclo de vida dos mesmos".

De acordo com Knight (1991), o estudo da confiabilidade surge após a Primeira Guerra Mundial, quando foi utilizado para descrever estudos comparativos feitos em aviões com um, dois ou quatro motores, adquirindo um significado tecnológico. Obteve avanços impulsionados pela indústria bélica durante e após a Segunda Guerra Mundial, na qual permitiram o amadurecimento da teoria. A partir de 1970 este conceito vem sendo aplicado nas mais diversas áreas (FOGLIATTO; RIBEIRO, 2009).

Segundo os mesmos autores, a análise de confiabilidade é uma metodologia utilizada para descrever o comportamento de falhas. Em seu sentido mais amplo, a confiabilidade está associada à operação bem-sucedida de um produto ou sistema, na ausência de quebras ou falhas. Entretanto, é necessária uma análise quantitativa desta definição. Leemis (1995) a descreve como a probabilidade de um determinado item desempenhar de forma adequada seu propósito especificado, por um determinado período de tempo, sob condições de operação estabelecidas. Desta forma a confiabilidade é definida como uma probabilidade.

Os principais conceitos relacionados à confiabilidade descritos pelas normas NBR ISO8402 (ASSOCIAÇÃO BRASILEIRA DE NORMAS TÉCNICAS, 1994) são: qualidade, disponibilidade, mantenabilidade, segurança e confiança.

Fogliatto e Ribeiro (2009) destacam a função de confiabilidade $R(t)$, a função de risco ou de falha $h(t)$ e o tempo médio até a falha MTTF, como sendo as medidas mais utilizadas em confiabilidade para unidades (componente, subsistema ou sistema), não sujeitas a reparos.

A função de confiabilidade $R(t)$ indica a probabilidade de a unidade apresentar sucesso na operação, ou seja, sem ocorrência de falhas no intervalo de tempo $(0, t)$ e ainda estar funcionando no tempo $t$. Em suma, a unidade 
deve cumprir sem falhas uma missão com uma duração determinada

A função de risco $h(t)$ é a medida de confiabilidade mais difundida na prática, ela interpreta a quantidade de risco associada a uma unidade no tempo $t$. Também conhecida como taxa de falha ou taxa de risco é extremamente útil na análise de falhas num processo interativo cujo sucesso depende de se determinar relações implícitas entre causa e efeito (CARVALHO, 2008).

O tempo médio até a falha MTTF (mean time to failure) define uma média dos tempos até ocorrer uma falha na unidade observada em questão.

A determinação da probabilidade de sobrevivência de uma unidade, até um determinado tempo $t$, é possível através da modelagem dos tempos até a falha. Esta é feita conhecendo-se a distribuição de probabilidade que melhor se ajusta a estes tempos. A partir delas pode-se determinar a probabilidade de sobrevivência da unidade para qualquer tempo $t$, o tempo médio até a falha e a função risco $h(t)$ do equipamento (FOGLIATTO; RIBEIRO, 2009).

Algumas distribuições podem ser utilizadas para representar a probabilidade de falha. As distribuições de probabilidade frequentemente utilizadas para descrever tempos até falha de componentes e sistemas são: Weibull, Lognormal, Gama e Exponencial (HAVIARAS, 2005).

Segundo Silva et al (2015), para realização da aplicação da confiabilidade é necessário um conhecimento do produto ou equipamento, pois é através deste conhecimento que será possível determinar os modos e as causas de falhas.

O comportamento de determinado produto com relação à falha é melhor compreendido pelo estudo de tempo de falha e exame do comportamento de sua taxa de falha (COLOSSIMO; FREITAS, 1997). A modelagem dos tempos até a falha é, portanto, central em estudos de confiabilidade (FOGLIATTO; RIBEIRO, 2009). Vários estudos estão sendo desenvolvidos com esse enfoque (DUEK, 2005; HAVIARAS, 2005; SANTOS et al, 2017; SILVA et al, 2015; SILVA et al, 2017).

A proposta desse artigo foi realizar uma análise quantitativa de confiabilidade de um inspetor eletrônico de garrafas, utilizado em indústrias de bebidas, utilizando os conceitos de análise de confiabilidade paramétrica.

\section{MATERIAIS E MÉTODOS}

$\mathrm{O}$ procedimento de pesquisa empregado no presente trabalho foi o estudo de caso de um equipamento eletrônico.

Os dados modelados neste estudo foram fornecidos pela empresa Ambev (Companhia de Bebidas das Américas). Estas informações foram recolhidas ao longo de uma semana de vistorias feitas ao equipamento. Trata-se de um inspetor eletrônico de garrafas utilizado para detectar a presença de objetos estranhos de baixa densidade, rachaduras, anéis de ferrugem, sujeira no acabamento do frasco e defeitos na rosca. Um equipamento extremamente importante na linha de produção. As falhas ocorridas foram geradas por dispositivos mecânicos transportadores 
responsáveis por carrear as garrafas através da máquina. Mais informações sobre o equipamento podem ser encontradas em Heuft (2017). Os tempos até as falhas, em minutos, estão apresentados na Tabela 1 .

Tabela 1 - Tempos até a falha.

\begin{tabular}{lllll}
\hline \multicolumn{5}{c}{ Tempos coletados em minutos } \\
\hline 3,2998 & 1,4666 & 1,4666 & 2,6665 & 4,2998 \\
3,7998 & 4,2927 & 3,6698 & 6,6330 & 1,0333 \\
4,6000 & 8,3000 & 0,5000 & 0,7000 & 8,0998 \\
3,0666 & 3,2664 & 6,4663 & 4,0331 & 5,8665 \\
0,9333 & 5,9333 & 1,2666 & 4,6000 & 0,7332 \\
0,2333 & 1,5000 & 2,6999 & 2,2239 & 1,5666 \\
1,6610 & 4,8392 & 5,4998 & 4,9039 & 1,4000 \\
4,8000 & 2,7332 & 6,3012 & 2,9655 & 4,4332 \\
& 2,0666 & 8,0665 & 2,6333 & \\
\hline
\end{tabular}

Fonte: Ambev.

Os dados disponibilizados foram trabalhados no ProConf (FRITSCH; RIBEIRO, 1998), um programa computacional projetado para o ajuste de distribuições de tempos de falha para dados de confiabilidade, através do uso de métodos gráficos e métodos analíticos. Além disso, fornece as estimativas dos parâmetros da distribuição e seus respectivos intervalos de confiança, e cálculos do tempo médio até a falha, taxa de risco e confiabilidade.

Os histogramas de frequência das falhas e os papéis de probabilidades foram utilizados como métodos gráficos de comparação com as curvas das distribuições conhecidas, a fim de se obter uma ideia inicial de qual modelo proporciona um melhor ajuste, ou seja, qual apresenta melhor aderência aos dados amostrais.

Para a caracterização da distribuição de frequência foram aplicados testes de aderência às distribuições de probabilidades. Os métodos analíticos utilizados para verificar o ajuste destas distribuições candidatas aos dados efluentes foram o teste paramétrico do Qui-Quadrado $\left(\chi^{2}\right)$ e o teste de Kolmogorov-Smirnov (K-S). Para cada teste, o software informa o nível de significância e aponta quais distribuições não podem ser rejeitadas (FRITSCH; RIBEIRO, 1998). A validação é dada se o nível de significância for maior que $5 \%$, em ambos os testes de aderência. Caso mais do que uma distribuição não possa ser rejeitada, cabe ao pesquisador, justificar a escolha por uma delas por fundamentação teórica.

A análise dos dados, utilizando os métodos descritos anteriormente, foi realizada para as distribuições exponencial, gama, Weibull e lognormal. Posteriormente foram feitas as estimativas dos parâmetros da distribuição da função distribuição de probabilidade que melhor modelou o conjunto de dados em estudo e apresentadas as representações das funções de confiabilidade $R(t)$ e de risco ou taxa de falha $h(t)$. 


\section{RESULTADOS E DISCUSSÃO}

Inicialmente foram obtidos alguns gráficos referentes ao conjunto de dados de tempo até a falha do inspetor eletrônico de garrafas. A frequência de ocorrência dessas falhas, número de falhas por tempo, está apresentada na Figura 1.

Figura 1 - Histograma dos tempos até falha.

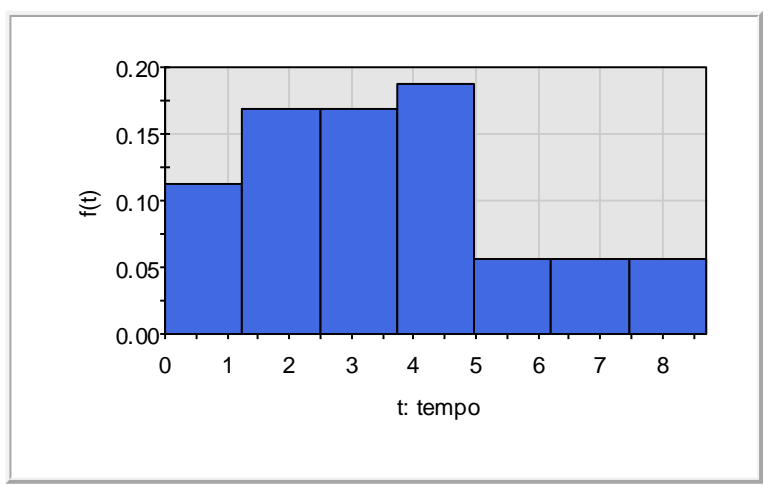

Fonte: ProConf.

Na Figura 2 está representada a taxa de falha ou de risco para o conjunto amostral.

Figura 2 - Taxa de Falha.

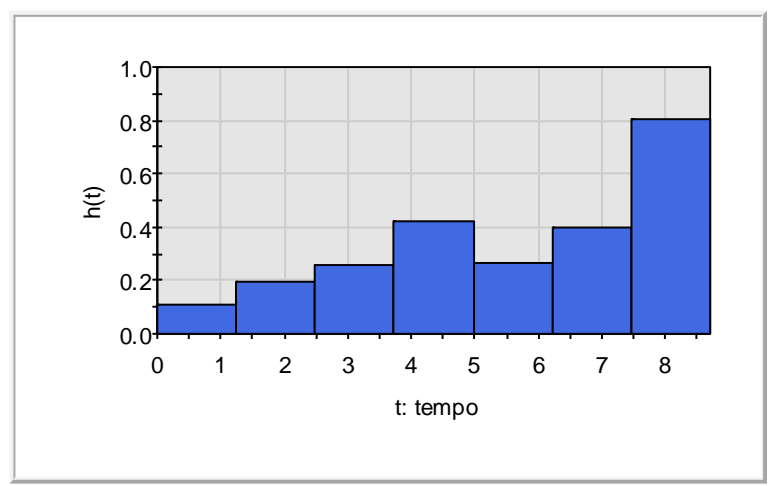

Fonte: ProConf.

Da Figura 2, observa-se que os dados apresentam uma relação inicialmente crescente, evidenciando uma elevação bem caracterizada da taxa de crescimento após 4 minutos.

O próximo passo foi verificar o ajuste dos distribuições de tempos de falha para a amostra em estudo, comparando o histograma dos tempos até a falha com as funções de probabilidade das distribuições. As análises gráficas indicam que as distribuições Weibull e lognormal poderiam ser candidatas a modelar adequadamente o conjunto de dados estudados, conforme Figuras 3 e 4 .

Figura 3 - Função densidade de probabilidade da distribuição Weibull.

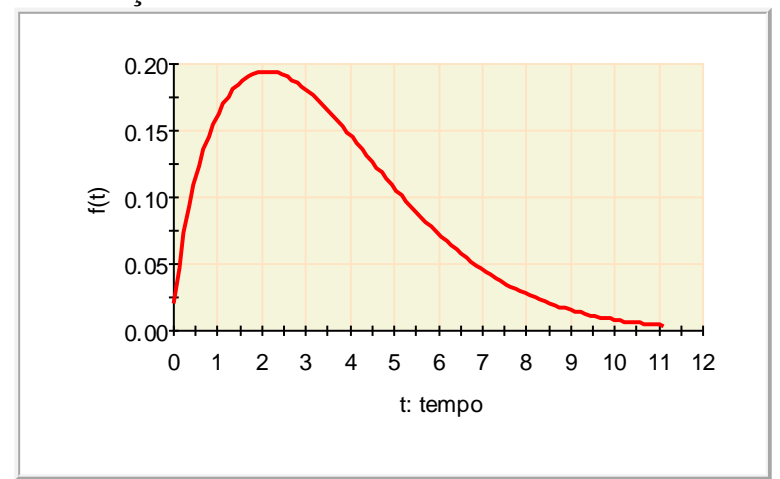

Fonte: ProConf.

Figura 4 - Função densidade de probabilidade da distribuição lognormal.

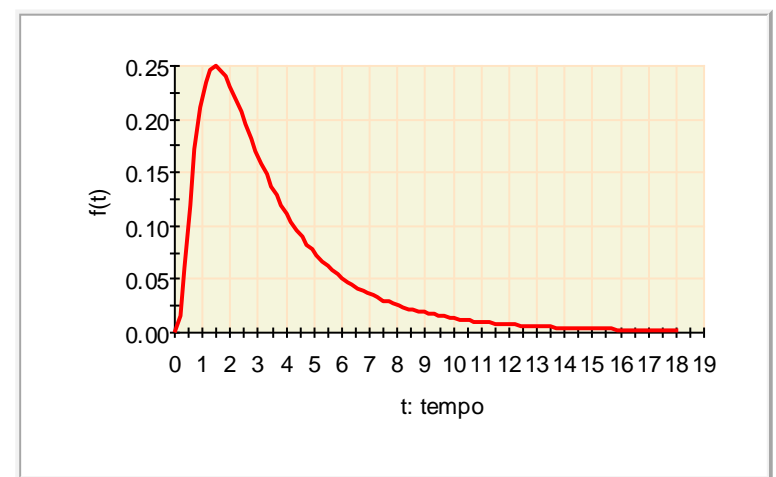

Fonte: ProConf.

Observa-se pelos gráficos da função densidade de probabilidade das distribuições, um melhor ajuste da distribuição Weibull.

Os gráficos do papel de probabilidade permitem fazer um teste de hipóteses, onde se vê a adequação dos dados a determinada distribuição. Nas Figuras 5 e 6 têm-se os aspectos do papel de probabilidade correspondentes às distribuições lognormal e Weibull. 
Figura 5 - Papel de probabilidade para a distribuição lognormal.

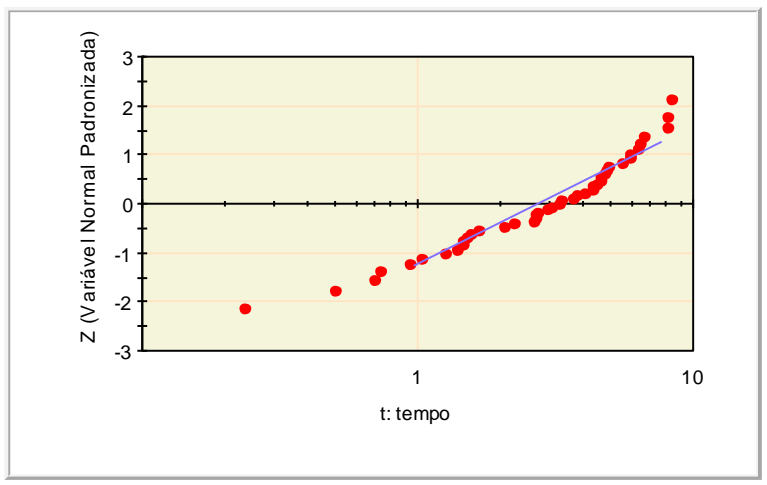

Fonte: ProConf.

Figura 6 - Papel de probabilidade para a distribuição Weibull.

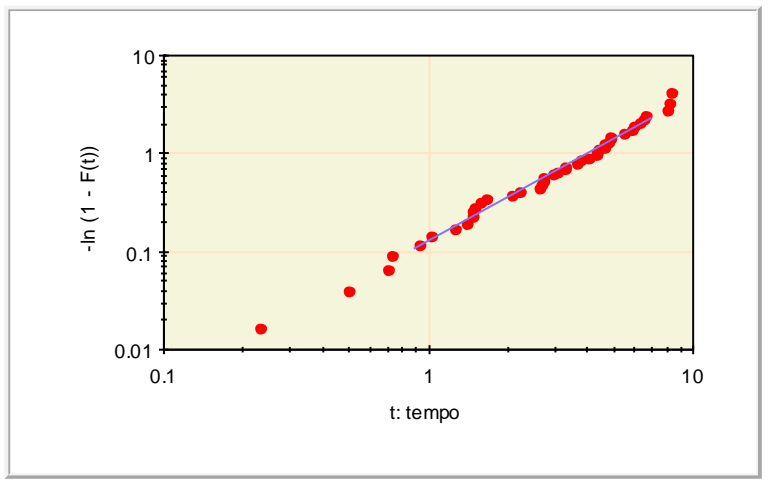

Fonte: ProConf.

Pela análise visual dos gráficos a distribuição que melhor modelou os dados foi a Weibull, nela os pontos estão visivelmente seguindo ao redor da reta-base, sem maiores dispersões, conforme Figura 6.

Na Tabela 2 estão apresentados os níveis de significância para os testes de aderência de Qui-Quadrado $\left(\chi^{2}\right)$ e do Kolmogorov-Smirnov $(K-S)$.

Tabela 2 - Níveis de significância dos testes de aderência.

\begin{tabular}{cccc}
\hline Modelo & $\chi^{2}$ & $K-S$ & Hipótese \\
\hline Exponencial & 0,0239 & 0,0016 & Rejeitada \\
Weibull & 0,7276 & 0,2768 & Não pode ser rejeitada \\
Gama & 0,5820 & 0,2518 & Não pode ser rejeitada \\
Lognormal & 0,1673 & 0,0573 & Não pode ser rejeitada \\
Normal & 0,3898 & 0,0156 & Rejeitada \\
\hline
\end{tabular}

Fonte: ProConf.
Observa-se a rejeição das hipóteses de que as distribuições exponencial e normal modelassem a amostra de dados. Para que os modelos fossem aceitos seria necessário que cada teste atingisse níveis de significância maiores que 0,05 , em ambos os testes.

Os demais modelos atingiram níveis superiores a $5 \%$ de significância e não puderam ser rejeitados. Os níveis destes modelos foram então comparados, concluindo-se que a distribuição Weibull obteve os melhores resultados. A partir destas evidências, o presente estudo utilizou a distribuição de Weibull para a modelagem do tempo até a falha. Fogliatto e Ribeiro (2009) afirmam que supor dados seguindo uma distribuição de Weibull costuma ser um bom ponto de partida na análise.

A distribuição Weibull é uma das distribuições mais importantes na modelagem de confiabilidade, devido à sua flexibilidade e capacidade de reprodução de amostras de tempos até a falha com comportamentos distintos (LAFRAIA, 2001; SANTOS et al, 2017; SILVA et al, 2017), além de poder ser usada em pequenas amostras (DODSON, 1994; FOGLIATTO e RIBEIRO, 2009; LEWIS, 1996).

Com a definição do modelo a ser seguido foi possível estimar seus parâmetros, bem como outros resultados dos ajustes apresentados pelo ProConf, conforme Tabela 3.

O $t_{10}$ e $t_{50}$ correspondem aos valores limites de tempos, nos quais $10 \%$ e $50 \%$ das falhas ocorreram. São apresentadas também as estimativas dos parâmetros da Weibull, representados por $\gamma$ e $\theta$. 
Tabela 3 - Resultados dos ajustes.

\begin{tabular}{cc}
\hline Parâmetro & Resultados \\
\hline$t_{10}$ (horas) & 0,95 \\
$t_{50}$ (horas) & 3,10 \\
MTTF (horas) & 3,50 \\
$\gamma$ & 1,64 \\
$\theta$ & 3,93
\end{tabular}

Fonte: Adaptado do software ProConf.

De acordo com a Tabela 3, o tempo médio até a falha (MTTF) dos inspetores é de 3,5 minutos. Metade dos equipamentos falham antes de 3,1 minutos. Além disso, os parâmetros de forma $\gamma$ e escala $\theta$ da Weibull são, respectivamente, 1,64 e 3,93.

O comportamento da taxa de falha de um equipamento ao longo do tempo pode ser analisado pela curva da banheira, que apresenta três períodos característicos de vida de componentes e equipamentos: mortalidade infantil, fase de maturidade, e mortalidade senil (SELLITTO, 2005). Cada fase da curva está associada ao parâmetro de forma da distribuição de Weibull (LAFRAIA, 2001; SELLITTO, 2005). A Tabela 4 descreve o comportamento da função de risco $h(t)$ segundo o parâmetro de forma $\gamma$.

Tabela 4 - Comportamento da função de risco $h(t)$.

\begin{tabular}{cc}
\hline$\gamma$ & Comportamento da função $h(t)$ \\
\hline$<1$ & Taxa de falha decrescente \\
$=1$ & Taxa de falha constante \\
$>1$ & Taxa de falha crescente \\
\hline
\end{tabular}

Fonte: Adaptado de Lafraia (2001).

Com base nos resultados, pode-se assumir que a fase de vida da amostra dos inspetores eletrônicos de garrafas estudados corresponde ao início da mortalidade senil, já que o parâmetro $\gamma$ da distribuição de Weibull foi maior que um.

As funções de risco (falha) e de confiabilidade são representadas nas Figuras 7 e 8 , respectivamente.

Figura 7 - Gráfico da função de risco $h(t)$.

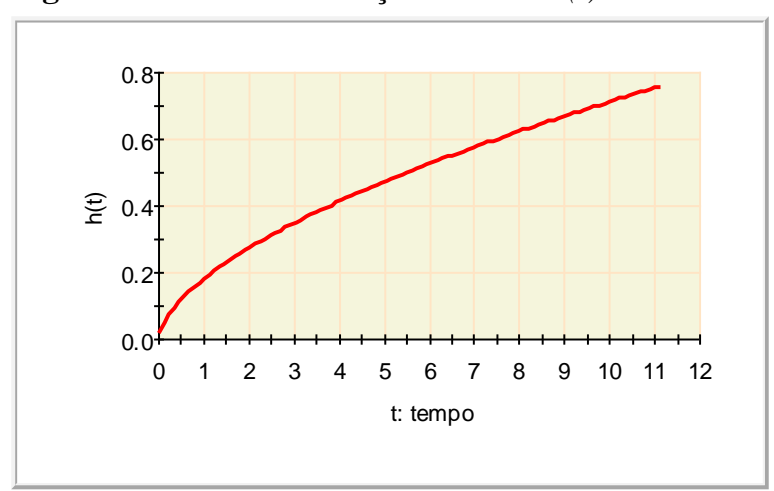

Fonte: ProConf.

Figura 8 - Gráfico da função Confiabilidade $R(t)$.

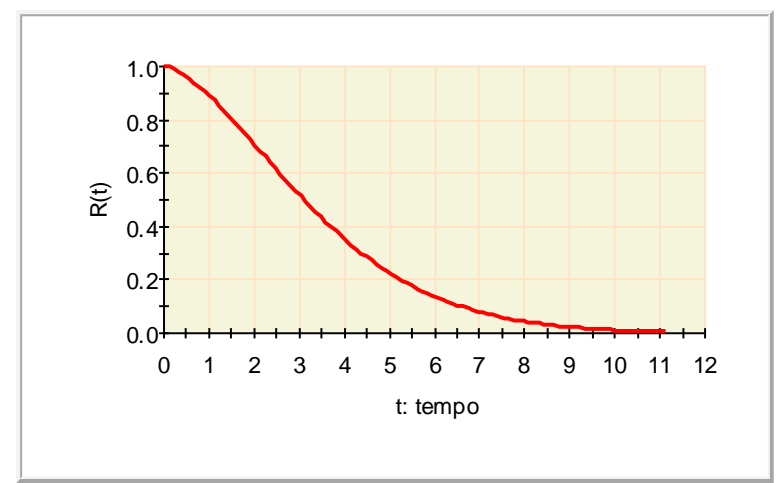

Fonte: ProConf.

Pode-se observar que a taxa de falha é crescente, cuja origem decorre do próprio desgaste natural do equipamento por funcionar durante um determinado tempo em sua vida útil. Nesta fase, as falhas tendem a ter defeitos cumulativos. $\mathrm{O}$ aumento muito rápido da taxa de falha, normalmente fundamenta o critério de quando peças devem ser substituídas e também determina a vida útil do produto ou sistema (SANTOS et al, 2017; SILVA et al, 2017). A estratégia para esta fase é a manutenção preventiva: a troca antecipa a inevitável quebra (SELLITTO, 2005). 
Em consequência, a função de confiabilidade $R(t)$ se comporta de maneira inversa, decrescente, ou seja, a confiabilidade do equipamento tende a diminuir ao longo do tempo.

\section{CONSIDERAÇÕES FINAIS}

Por meio de estudos quantitativos de confiabilidade é possível estabelecer parâmetros capazes de auxiliar de maneira precisa a tomada de decisão referentes a realização de investimentos, estudos de garantia e procedimentos de manutenção e operação com maior embasamento científico.

Por meio de métodos gráficos e analíticos, definiu-se a distribuição Weibull como a que melhor se ajusta aos dados estudados. Foi possível obter as medidas de confiabilidade do tempo médio até a falha, valores limites de tempo (sendo que nestes $10 \%$ e $50 \%$ das falhas ocorreram), bem como estimar os parâmetros da distribuição ajustada.

A expectativa de tempo até a falha dos itens em estudo foi de 3,50 minutos e metade deles falharam antes 3,10 minutos.

Analisando os resultados conclui-se que os dispositivos transportadores do inspetor eletrônico de garrafas já atingiram a fase de envelhecimento, ou seja, a fase final de sua vida, mostrando risco crescente e confiabilidade decrescente, indicando um desgaste do equipamento.

Por fim estas análises quantitativas dos tempos até as falhas do equipamento são relevantes para a determinação de estratégias de manutenção a ser realizadas pela empresa, a fim de garantir maior disponibilidade do produto.

\section{REFERÊNCIAS}

ASSOCIAÇÃO BRASILEIRA DE NORMAS TÉCNICAS. NBR ISO 8402. Gestão da qualidade e garantia da qualidade - terminologia. 1994.

CARVALHO, Alessandra Lopes. Análise de Disponibilidade Utilizando Abordagem Nebulosa. 2008. 123f. Tese (Doutorado). Universidade Federal de Minas Gerais, Departamento de Engenharia Elétrica, Belo Horizonte.

COLOSIMO, E. A.; FREITAS, M. A.

Confiabilidade: análise de tempo de falha e testes de vida acelerados. Belo Horizonte: QFCO, 1997. $309 \mathrm{p}$.

DODSON, B. Weibull Analysis. Milwaukee: ASQ, 1994.

DUEK, C. Análise de confiabilidade na manutenção de componente mecânico de aviação. 2005.

Dissertação (Mestrado em Engenharia de Produção) Universidade Federal de Santa Maria, Brasil.

FOGLIATO, F. S.; RIBEIRO, J L. D. Confiabilidade e Manutenção Industrial. Elsevier, Rio de Janeiro, 2009.

FRITSCH, C.; RIBEIRO, J. ProConf: Um software orientado para análises de confiabilidade. In. XVIII Encontro Nacional de Engenharia de Produção. Niterói, 1998.

HAVIARAS, Gilberto. J. Metodologia para análise de confiabilidade de pneus radiais em frota de caminhões de longa distância. 2005. 124 p. Dissertação (Mestrado em Engenharia Automotiva), Escola Politécnica da Universidade de São Paulo, São Paulo, 2005.

HEUFT, InLine. Inspetor Eletrônico de Garrafas. Disponível em:

<https://heuft.com/en/product/beverage/emptycontainers/empty-bottle-inspection-heuft-inline>. Acesso em: 15 nov. 2017.

LEEMIS, L. Reliability: probabilistic models and statistical methods. Nova York: Prentice-Hall, 384p., 1995.

LEWIS, E. Introduction to reliability engineering. New York: John Wiley \& Sons, 1996. 
MARCORIN, A. J.; ABACKERLI, A. J. Estudo exploratório sobre áreas potenciais de aplicação de técnicas de confiabilidade. In: Encontro Nacional de Engenharia de Produção, XXI, 2001, Salvador. Anais. Porto Alegre: ABEPRO, 2001, 1 CD-ROM.

MENDES, A. A. Manutenção centrada em confiabilidade: uma abordagem quantitativa. 2011. Dissertação (Mestrado em Engenharia) -

Universidade Federal do Rio Grande do Sul, Brasil.

KNIGHT, C.R. Four decades of reliability progress. Proceeding of the Annual Reliability and Maintainability Symposium. [S.1.]: IEEE Reliability Society, 1991. P.156-159.

SANTOS, M. M. M.; SILVA, E. C.; FERREIRA, G. S.; ROSAS, R. M. G.; ANDRADE, P. C. R.

Modelagem do tempo de vida de um inversor de frequência. ForScience: revista científica do IFMG, Formiga, v. 5, n. 3, e00288, 2017.

SELLITTO, M. Formulação estratégica da manutenção industrial com base na confiabilidade dos equipamentos. Produção, v.15, n.1, p.044-059, 2005.

SILVA, J. R. S.; SOUZA, L. A. D.; CASTRO, L. Z.; FERREIRA, T. A.; CAMPOS, M. S.Análise da Confiabilidade: Um estudo de caso. In. XXXV Encontro Nacional de Engenharia de Produção, Fortaleza, 2015.
SILVA, E. C.; FERREIRA, G. S.; SANTOS, M. M. M.; ANDRADE, P. C. R.; ROSAS, R. M. G. Análise de Dados de Falha de um Transmisor de Fibra Óptica. Revista Thema, Pelotas, v. 14, n. 4, 2017, p. 259 a 266.

Ellen Maria Neves Silva

Bacharelado em Ciência e Tecnologia - UFVJM.

\section{Paulo César de Resende Andrade}

Professor Associado do Instituto da Ciência e Tecnologia da Universidade Federal dos Vales do Jequitinhonha e Mucuri - UFVJM 